Published in final edited form as:

Pediatr Infect Dis J. 2019 June ; 38(6): 595-599. doi:10.1097/INF.0000000000002205.

\title{
Community Origins and Regional Differences Highlight Risk of Plasmid-Mediated Fluoroquinolone Resistant Enterobacteriaceae Infections in Children
}

Latania K. Logan, MD, MSPH ${ }^{1,2,9,10}$, Rachel L. Medernach, MD ${ }^{1,8}$, Jared R. Rispens, MD $^{1,8}$, Steven H. Marshall, MS $^{2}$, Andrea M. Hujer, BS ${ }^{2,3}$, T. Nicholas Domitrovic, MS ${ }^{2,3}$, Susan D. Rudin, BS ${ }^{2,3}$, Xiaotian Zheng, MD, PhD ${ }^{5,6}$, Nadia K. Qureshi, MD ${ }^{7}$, Sreenivas Konda, PhD ${ }^{9}$, Mary K. Hayden, MD ${ }^{8}$, Robert A. Weinstein, MD ${ }^{8,10}$, and Robert A. Bonomo, MD ${ }^{2,3,4}$

${ }^{1}$ Pediatrics, Rush University Medical Center, Chicago, Illinois, United States

${ }^{2}$ Research Service, Louis Stokes Cleveland Department of Veterans Affairs Medical Center, Cleveland, Ohio

${ }^{3}$ Medicine, Case Western Reserve School of Medicine, Cleveland, Ohio

${ }^{4}$ Pharmacology, Molecular Biology, and Microbiology, Case Western Reserve School of Medicine, Cleveland, Ohio

${ }^{5}$ Microbiology, Ann \& Robert H. Lurie Children's Hospital of Chicago, Chicago, Illinois, United States

${ }^{6}$ Pathology, Northwestern Feinberg School of Medicine, Chicago, Illinois, United States

${ }^{7}$ Pediatrics, Loyola University Medical Center, Maywood, Illinois, United States

${ }^{8}$ Medicine, Rush University Medical Center, Chicago, Illinois, United States

9 University of Illinois at Chicago, Chicago, Illinois

${ }^{10}$ Cook County Health and Hospital Systems, Chicago, Illinois, United States

\section{Abstract}

Background: Fluoroquinolones (FQs) are uncommonly prescribed in children, yet pediatric multidrug-resistant (MDR)-Enterobacteriaceae (Ent) infections often reveal FQ resistance (FQR). We sought to define the molecular epidemiology of FQR and MDR-Ent in children.

Methods: A case-control analysis of children with MDR-Ent infections at 3 Chicago hospitals was performed. Cases were children with third-generation-cephalosporin-resistant (3GCR) and/or carbapenem-resistant (CR)-Ent infections. PCR and DNA analysis assessed bla and plasmidmediated FQR (PMFQR) genes. Controls were children with 3GC, FQ and carbapenem

Corresponding Author: Latania K. Logan, Rush University Medical Center 1620 W. Harrison, Suite 951 Jelke, Chicago, IL 60612; Phone 312-942-8928; Latania_Logan@ rush.edu.

Conflicts of Interest: The authors have no conflicts of interest relevant to this article.

Financial Disclosure: The authors have no financial disclosures relevant to this article. 
susceptible-Ent infections matched by age, source and hospital. We assessed clinicalepidemiologic predictors of PMFQR Ent infection.

Results: Of 169 3GCR and/or CR Ent isolates from children (median age 4.8 years), 85 were FQR; 56 (66\%) contained PMFQR genes. The predominant organism was E. coli and the most common bla gene bla ${ }_{\mathrm{CTX}} \mathrm{M}-1$ group. In $\mathrm{FQR}$ isolates, $\mathrm{PMFQR}$ gene mutations included aac6' $1 b$-cr, oqX $A / B, q e p A$, and $q n r A / B / D / S$ in $83 \%, 15 \%, 13 \%$ and $11 \%$ of isolates, respectively. FQR E. coli was often associated with phylogroup B2, ST43/ST131. On multivariable analysis, PMFQR Ent infections occurred mostly in outpatients (OR 33.1), and those of non-black-white-Hispanic race (OR 6.5). Residents of Southwest Chicago were $>5$ times more likely to have PMFQR-Ent infections than those in the reference region, while residence in Central Chicago was associated with a 97\% decreased risk. Other demographic, comorbidity, invasive-device, antibiotic use, or healthcare differences were not found.

Conclusions: The strong association of infection with MDROs showing FQR with patient residence rather than with traditional risk factors suggests that the community environment is a major contributor to spread of these pathogens in children.

\section{Keywords}

Epidemiology; Gram-negative bacteria; Enterobacteriaceae infections; drug resistance; children

\section{Introduction}

Multi-drug resistant (MDR) Enterobacteriaceae infections are associated with significant morbidity and mortality and are an emerging problem in children in the US during the last decade $(1,2)$. Globally, this has been attributed mainly to the rise in extended-spectrum beta lactamase (ESBL) producing Enterobacteriaceae (ESBL Ent) and carbapenem-resistant Enterobacteriaceae (CRE) (3, 4). Increasing resistance to several other classes of antibiotics was found in these studies; including to the fluoroquinolones, a class of antibiotics with limited indications for use in children $(1,2)$.

The CTX-M-ESBL harboring Escherichia coli are among the most common multi-drug resistant organisms (MDROs), often possessing resistance genes to other important antibiotic classes including aminoglycosides, fluoroquinolones, tetracyclines, and trimethoprim-sulfamethoxazole (5). In adults, fluoroquinolone resistance in (FQR) Gramnegative bacteria has been linked to chromosomal and to plasmid-mediated resistance mechanisms and is thought to be associated mostly with the dramatic increase in use of these antibiotics during the $1980 \mathrm{~s}(6)$.

The reasons for increased FQR in children are unclear, and studies assessing the resistance determinants associated with the FQR phenotype in Enterobacteriaceae recovered from children are limited(7); therefore, we examined this population to determine which children had a higher likelihood of infections with organisms resistant to both beta-lactam and fluoroquinolone antibiotics.

We determined the genetic basis of $\mathrm{FQR}$ in beta-lactamase producing Enterobacteriaceae isolates from children cared for by multiple centers in the Chicago area and analyzed a 
subgroup of children with infections with similar resistance determinants, namely those due to isolates containing genes encoding plasmid-mediated fluoroquinolone resistance (PMFQR) and ESBL mediated resistance to determine genotypes, host factors, and exposures leading to infection with MDR Enterobacteriaceae strains. We hypothesized that because of differences in healthcare delivery in urban settings, acquisition of PMFQR in children would be linked to geographic location and have environmental influences and community origins. Our analysis of host factors and exposures revealed that there are genetic and geospatial links to MDR in this pediatric population.

\section{METHODS}

\section{Study Setting}

Hospital A contains a 115-bed children's hospital within a tertiary care academic medical center which has a mother-newborn infant unit, pediatric and psychiatric wards, and cardiac, pediatric and neonatal intensive-care units (PICU and NICU). Hospital B has 288 beds and is a free-standing children's academic medical center that provides complex quaternary services, such as pediatric organ and bone marrow transplantation. Hospital C is a 125-bed children's hospital within an academic medical center, and contains general pediatrics and newborn infant wards, as well as a PICU and NICU. All of the participating centers are within metropolitan Chicago.

\section{Descriptive Study Design}

Study Population-This study included patients aged 0 to 18.99 years who had clinical cultures positive for Enterobacteriaceae with 3GCR or CR and the suspected presence of a beta-lactamase gene based on clinical laboratory testing. Additionally, isolates found to be concomitantly resistant to $\mathrm{FQR}$ were further characterized. Infections were diagnosed between January 1, 2011 and December 31, 2014 and only the first infection per patient was included. The study was approved by the institutional review boards of the three participating institutions and need for informed consent was waived.

\section{Testing of Antibiotic Susceptibility in Enterobacteriaceae}

The Hospitals A-C microbiology laboratories phenotypically analyzed presumed ESBL Ent, AmpC Ent and CR isolates via the Vitek 2 microbial identification system (bioMérieux, Athens, GA) or by the MicroScan WalkAway system (Siemens Healthcare Diagnostics, Tarrytown, NY). Screening for ESBL production involved testing with one or more of the following agents: aztreonam, ceftazidime, ceftriaxone, cefotaxime or cefpodoxime, based on guidelines of the Clinical and Laboratory Standards Institute (CLSI) (8). ESBL production was confirmed on the automated instruments or by disk diffusion assays (BBL; Becton, Dickinson and Company, Sparks, MD) or by measuring minimum inhibitory concentrations (MICs) of ceftazidime and cefotaxime in the presence and absence of clavulanic acid. A measurement of an increase in disk zone diameter of $>5 \mathrm{~mm}$ or a 4-fold reduction in the MIC of ceftazidime or cefotaxime in the presence of clavulanic acid served as confirmation of the ESBL phenotype (8). 
The carbapenemase phenotype, per Centers for Disease Control and Prevention (CDC) criteria, included isolates that were non-susceptible to all 3GCs (cefotaxime, ceftazidime, or ceftriaxone) and resistant to one or more carbapenem (imipenem, meropenem, doripenem, or ertapenem) (9). Carbapenemase production was phenotypically confirmed by MBL E-test (bioMérieux, Athens, GA) or Modified Hodge Test, as appropriate (10).

\section{Determination of Beta-Lactam Resistance Mechanisms}

Genomic DNA was extracted and purified from isolates using the DNeasy Blood \& Tissue Kit (QIAGEN, Inc., Valencia, CA). To evaluate for the presence of bla genes in isolates, a DNA microarray based assay was performed (Check-Points, Check-MDR CT101 kit; Wageningen, The Netherlands). The CT101 microarray based assay can detect the following bla groups: CTX-M-1 group, CTX-M-2 group, CTX-M-8 and -25 group, CTX-M-9 group, SHV WT and SHV-type ESBL, TEM wild-type, and TEM-type ESBL, plasmid based AmpC cephalosporinases (pAmpC) (CMY II, ACC, FOX, DHA, ACT/ MIR) and carbapenemases (KPC and NDM) (11). When isolates were bla negative by the CT101 assay, a broader DNA microarray, (Check-Points, Check-MDR CT103XL kit) was performed. The CT103XL assay can detect the presence of additional ESBL genes (VEB, PER, BEL, GES) and carbapenemase genes (GES, GIM, IMP, SPM, VIM, and OXA-23, $-24 / 40,-48$, and -58$)(12)$. The assays were performed as described in our laboratory previously (13).

\section{Analysis of Determinants Yielding Fluoroquinolone Resistance}

To investigate the presence of $\mathrm{FQR}$ determinants in MDR Enterobacteriaceae isolates, we analyzed the quinolone resistance-determining region (QRDR) located on the bacterial chromosome and assessed for PMFQR in strains found FQR by CLSI standards (8). Briefly, genus specific assays for mutations in $g y r A$ and parC genes of the QRDR (in E. coli, Klebsiella sp., and Enterobacter sp.) and for PMFQR were performed by polymerase chain reaction (PCR) and deoxyribonucleic acid (DNA) sequencing of amplicons (6). Extraction of genomic DNA followed by amplification and sequencing were performed using primers and methods as previously described $(14,15)$. Specific PMFQR genes screened include $q n r A, q n r B, q n r D$, qnrS, qepA, oqxA and $o q x B$ and aac6'-Ib-cr and represent transmissible elements reported in Enterobacteriaceae (16).

\section{Multilocus Sequence Typing (MLST)}

Per protocol, eight $E$. coli housekeeping genes ( $\operatorname{din} B, i c d A, p a b B, p o l B$, putP, $\operatorname{trp} A$, trpB and uidA) and seven Klebsiella species (sp.) housekeeping genes (rpoB, gapA, $m d h$, pgi, phoE, $\operatorname{ton} B$, inf $B$ ) were amplified and sequenced as in prior studies (13). Alleles and sequence types (ST) were assigned for select isolates of varying genotypic profiles by the Pasteur MLST scheme (http://www.pasteur.fr/recherche/genopole/PF8/mlst/).

\section{Analysis of Plasmid Replicon Types and Phylogenetic Grouping}

E. coli were assigned to four major phylogenetic groups (A, B1, B2 and D) using a wellestablished multiplex PCR-based method (17). Plasmids were typed, in select isolates with 
varying genotypic profiles, based on incompatibility groups corresponding to the nomenclature assigned by Carattoli et al. (18).

\section{Analytic Study Design}

We used a retrospective case-control study design to assess factors associated with infection due to beta-lactam, FQ resistant isolates in which we had detected a PMFQR gene. We chose to analyze FQR in detail because this class of antibiotics is uncommonly used in children, yet 50\% of the isolates between 2011 and 2014 were FQR.

We selected as controls, children with infections due to bacteria susceptible to the antibiotics of interest. Specifically, the control group included children with infections that were susceptible to $3 \mathrm{GC}$, carbapenem and FQ antibiotics to understand differences between children who acquired plasmid-mediated MDR Enterobacteriaceae infections and those who did not.

Only patients with clinical infections were included, as determined by study investigator case review and/or using standard criteria defined by the CDC National Healthcare Safety Network (19). Children serving as control subjects were identified using hospital electronic laboratory records (ELRs). Control patients were matched approximately 3:1 to the cases by age range, hospital, and specimen source.

\section{Covariates}

Several variables were analyzed as potential factors associated with FQR Ent infection based on known associations for acquisition in adults including (1) demographics (age, gender, race/ethnicity); (2) comorbid conditions (as defined by ICD-9 codes); (3) recent inpatient and outpatient healthcare exposures, including hospitalization and/or procedures in the previous 30 days; (4) all recent antibiotic exposures in the 40 days prior to culture (32); (5) presence, number, and type of invasive medical devices; and (6) the impact of location of patient residence in the Chicago area as assessed by dividing the metropolitan area into 7 regions using zip code level data, which included Chicago proper and its suburban areas (i.e. Northwest side and Northwest Suburbs, Southwest side and Southwest Suburbs, etc.). An eighth region included patients from other parts of Illinois or from other states.

\section{Statistical Analysis}

Case and control groups were examined for differences using parametric or non-parametric tests as appropriate for categorical and continuous variables; $\mathrm{P} \unlhd 0.05$ was considered statistically significant unless otherwise specified. Variables with $\mathrm{p}<0.1$ on bivariate analysis were included in multivariable analysis. Stepwise multiple logistic regression was used to assess the multivariable relationship between the covariates and the groups. The final multivariable logistic regression model included the simplest model with significant covariates $(\mathrm{p}<0.05)$ from the stepwise selection process, with PMFQR Ent infection as the outcome variable. The simplest model was chosen based on a relatively small sample size and the effect of variables in the model. All analyses were performed in SAS 9.4 (SAS Institute, Cary, NC, USA). 


\section{RESULTS}

\section{Composition of Fluoroquinolone Resistance (FQR) Genes in Enterobacteriaceae}

We assessed 169 bla-producing Ent isolates between 2011 - 2014 from Hospitals A, B, and $\mathrm{C}$ for the presence of FQR (Table 1). Of 169 Ent isolates, 85 (50\%) were FQR of which 82 (96.4\%) were available for further testing. The median age of children with FQR-Ent infections was 4.8 years. The predominant organism was E. coli, 65/82 (79\%), and the predominant bla genotype found associated with $\mathrm{FQR}$ in Ent was bla cases. Within E. coli, $\mathrm{FQR}$ was most often associated with phylogroup B2 and ST43 (Pasteur scheme)/ST131 (Achtman scheme) harboring bla $a_{\mathrm{CTX}}$-M-1-group in 47/63 (75\%) cases.

FQR isolates were further characterized to understand resistance determinants associated with FQR in pediatric Ent isolates. Chromosomal mutations of the QRDR ( $g y r A / p a r C)$ were present in 71/79 (89.9\%) of FQR isolates by DNA sequence analysis. Three isolates did not yield results. PMFQR genes were detected by PCR in 56/82 (66\%); 53 (95\%) were available for further analysis. The median case patient age was 6 years. PMFQR genes included aac 6' $1 b$-cr, oqx $A / B, q e p A$, and $q n r A / B / D / S$ in $83 \%, 15 \%, 13 \%$ and $11 \%$ of isolates, respectively. PMFQR was found in combination with $g y r A$ and/or parC mutations in 43/49 $(88 \%)$ isolates, which is associated with high level resistance. The predominant bla genotype found associated with PMFQR was bla associations in 11\%. Almost all (98\%) PMFQR Ent were multi-drug resistant, e.g. resistant to $>3$ antibiotic classes.

\section{Analysis of Factors Associated with PMFQR Enterobacteriaceae Infections in Children}

The 53 cases of PMFQR Ent infection were matched by age range, hospital, and culture source to 131 controls with antibiotic-sensitive Ent infections. Significant factors associated with PMFQR Ent infection on bivariate analysis included: E. coli infection, race/ethnicity, infection diagnosed in an outpatient clinic, history of quinolone use, and residence in the Southwest region (hereafter referred to as the high-risk region) comprised of southwest Chicago and the southwestern Chicago suburbs (see Table, Supplemental Digital Content 1). Children with PMFQR infection were less likely to have infection with Enterobactersp., a central venous catheter, neonatal intensive care unit admission at the time of infection diagnosis, or residence outside the "high-risk" region (comprised of the downtown Chicago area, near North side, Chicago loop, and North Chicago). Case-control differences in comorbid conditions; presence of respiratory, gastrointestinal, genitourinary, or overall count of foreign bodies; or recent prior healthcare exposure were not found.

We did not find evidence of significant effect modification during the model building stages nor did we find evidence of significant confounding; therefore, no additional covariates were added back to the final model after the stepwise selection process was completed, and the simplest model was used in the final regression model.

On multivariable analysis (Table 2), having infection diagnosed in the outpatient clinic setting was significantly associated with PMFQR Enterobacteriaceae infection (OR=33.1; 95\% CI 7.1, 162.8; $\mathrm{p}<0.001)$. Being of a race or ethnicity other than white, black, or 
Hispanic was significantly associated with PMFQR Enterobacteriaceae infection (OR 6.5; 95\% CI 1.7, 24.3; p=0.006). Interestingly, among children with Enterobacteriaceae infections, those residing in southwestern region of Chicago had more than five times the odds of having a PMFQR infection compared to those living in the reference West Chicago region (OR 5.6; 95\% CI 1.6, 19.2; $\mathrm{p}=0.006$ ) after controlling for race and healthcare setting. In contrast, for children who resided in the downtown region, there was a $97 \%$ decrease in the odds of PMFQR infection in those living in this region compared to those residing in reference West Chicago region (OR 0.03; 95\% CI .002, 0.33; $\mathrm{p}=0.005$ ). No other regional associations were found.

To ensure these regional associations were unique to PMFQR containing isolates, we ran two additional case-control analyses specifically assessing whether regional differences within the Chicago metropolitan area were seen with 1) ESBL-producing strains that were

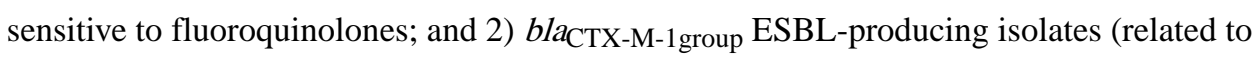
circulation of ST131 clonal E. coli strains). Regional differences in acquisition were not found in either analysis (data not shown).

\section{DISCUSSION}

Multi-drug resistant Enterobacteriaceae are a growing concern globally. Much of the propagation and spread of these organisms has been related to the ST131 E. coli strains and to high-risk clones containing IncFII plasmids and other genetic structures, such as transposons, integrons, and insertion sequences associated with multiple antibiotic resistance gene cassettes (20). Occasionally, beta-lactamase and other antibiotic resistance genes are transferred horizontally (7).

In our pediatric patients there was a predominance of ST131 E. coli harboring bla ${ }_{\mathrm{CTX}-\mathrm{M}}$. We hypothesize that there is significant horizontal gene transfer between genera. This is very worrisome from a public health perspective since children, once colonized with MDR Enterobacteriaceae can remain colonized for months to years and could serve as reservoirs and "silent disseminators" of MDROs (21). Interestingly, the community focus of the MDROs in children is in stark contrast to the epidemiology of these bacteria in adults in Chicago, where MDR Enterobacteriaceae acquisition is highly linked to residence in longterm care facilities and to interfacility transfer (22).

We found striking residential differences for children infected with PMFQR containing Enterobacteriaceae (PMFQR Ent) compared to children infected with antibiotic sensitive strains. PMFQR Ent contain plasmid-based resistance genes to fluoroquinolones, an antibiotic uncommonly used in children. In one Chicago region, the Southwest region, there was a substantial increase in odds of PMFQR Ent infection, and in the Downtown region, there was a significant decrease in the likelihood of PMFQR Ent infection. Our study included 3 major pediatric centers, none of which (and no major medical centers for children) is located in the "high-risk" Southwest region, yet all three centers diagnosed and treated patients with PMFQR Ent infections from that region. In contrast, Hospital B, the largest hospital in the region specifically dedicated to the care of children, is located in the downtown region, and therefore services many children residing in that area; yet this was the 
area of "lowest risk" for PMFQR Ent infection. This may reflect linkage to bla CTX-M harboring plasmids which are endemic in some communities, but the reservoirs are currently undefined.

Interestingly, we found that children with PMFQR Ent infection were more likely to present in the outpatient clinic setting than were those with antibiotic sensitive Ent infections - the opposite of expectation that MDROs are healthcare linked and suggesting that PMFQR Ent infections were community acquired and less severe.

A strong additional association on multivariable analysis was the higher likelihood of PMFQR Ent infection in those of non-white, non-black, and non-Hispanic race. This risk was statistically independent of residence, and strikingly none of the children located in the "high risk" southwest region with PMFQR Ent infection were of race "other", supporting the strong independence of these 2 risk factors. Due to the retrospective nature of the study, we were unable to gather further data on "other" race or ethnicity. We did not have travel data for the majority of children, although it is well documented that travel to certain countries can be associated with high rates of ESBL Ent acquisition, particularly in South and Southeast Asia $(23,24)$. It is also well described that there is an increased risk of colonization of household members after return of the traveler who first acquired an ESBL Ent.(25)

We did not find significant differences in comorbidities between cases and controls. This puts further importance on evaluating environmental sources for plasmid-possessing antibiotic resistance genes (26). Community-based environmental influences would include higher exposure risks in certain communities due to certain foods, livestock, animals, water sources, fertilizer, soil, and vegetation (27). For example, if there is a link to food exposures, such as restaurant chains that cook with high saturated fats, and additionally serve food animals that are fed antibiotics and hormones for growth effects, this exposure would increase the risk of acquisition of antibiotic-resistant bacteria, as well as obesity (28). This in turn increases the risk of other diseases such as cardiovascular disease and diabetes (29).

Some of the PMFQR genes, for example, oqxA and oqxB are multidrug efflux pumps named for their resistance to olaquinadox, which is used as a growth promoter on pig farms (30). We did not have data on companion pets for the majority of children and were unable to examine this association, though this may play an important role in acquisition of resistant pathogens (9).

Studies in our region and nationally have suggested that an increased risk of exposure to antibiotics in children (31), as well as to antibiotic resistant bacteria, may be related to socioeconomic status and race (32). While we did assess race, we did not formally compare differences between socioeconomic factors in the regions, as we did not have street or neighborhood level data on infected patients. However, in a general comparison of regional zip codes using Illinois census data, we did not find overall differences in the socioeconomic status of the "high risk" southwest region and neighboring regions such as the south and west regions. We did not have data on antibiotic usage in adults within the household, which could also increase acquisition of resistant pathogens. 
We recognize that our study has limitations. This was a retrospective study designed to determine mechanisms of antibiotic resistance in Enterobacteriaceae recovered from children cared for at three centers in a single metropolitan area; this may potentially impact generalizability to other regions. Additionally, a plasmid-based origin of the recovered antibiotic-resistance genes is suggested by our DNA sequence analysis results, yet it is possible that some of these genes represent chromosomal resistance mechanisms. However, subsequent plasmid-replicon typing and DNA sequence analysis for a subgroup of bacteria support our findings of the DNA microarray. Our sample size was relatively small, which may allow for selection bias; however, the pooling of multi-centered data from institutions of differing types serving diverse populations throughout the 3rd largest metropolitan area in the U.S. potentially lessens this bias. The smaller sample sizes in pediatric studies are related to the overall low prevalence of these organisms in children in most U.S. areas (1-3\%), including in the Chicago and the Midwest region $(21,23,26)$, although national trends indicate an increase in prevalence of these menacing organisms in pediatric populations during the last decade, suggesting they are an emerging threat that needs further evaluation (7).

In conclusion, we found that there is significant complexity and diversity in the determinants associated with beta-lactam and fluoroquinolone antibiotic resistance in children, and that pediatric MDR Enterobacteriaceae exhibited differences when compared to descriptions of strains circulating in adult patients in a region where such infections are endemic. We also describe, for the first time, the impact of residence on infection with MDR Enterobacteriaceae in children located in the same geographic area, however the reservoirs remain undefined. Future studies should focus on further molecular characterization of circulating strains and the environmental influences associated with these differences in regional acquisition. We anticipate that an imminent threat of the "silent dissemination" of multi-drug resistant Enterobacteriaceae in community settings is occurring in children. Local, federal, and international programs dedicated to this serious problem must focus on halting the spread of these menacing pathogens in our most vulnerable population, children.

\section{Supplementary Material}

Refer to Web version on PubMed Central for supplementary material.

\section{Acknowledgements}

We gratefully acknowledge the contribution of the late Dr. Paul Schreckenberger to this work.

We thank the microbiology laboratories of the participating institutions for providing isolates for this study. We thank Kendrick Reme and Lynika Strozier of the Logan Laboratory and Pamela Hagen, Jane Stevens, Joyce Houlihan, Kathleen McKinley, Violeta Rekasiu, Cindy Bethel, and Donna Carter of participating institutions for collection, shipping, and cultivation of organisms. We thank Drs. Ronald Hershow and Maria Argos for critical review and feedback of this work. We thank the team of curators of the Institut Pasteur MLST and whole-genome MLST databases for curating the data and making them publicly available at http://bigsdb.web.pasteur.fr/. We report no conflicts of interest relevant to this study.

The content is solely the responsibility of the authors and does not necessarily represent the official views of the National Institutes of Health or the Department of Veterans Affairs.

Funding 
This work, including the efforts of Latania K. Logan, was funded by the National Institute of Allergy and Infectious Diseases, National Institutes of Health (NIH) (K08AI112506). This work, including the efforts of Robert A. Bonomo, was funded by the National Institute of Allergy and Infectious Diseases, National Institutes of Health (NIH) (R01AI072219, R01AI063517, and R01AI100560). R.A.B. is also supported by the Department of Veterans Affairs Research and Development under award number I01BX001974, VISN 10 Geriatrics Research, Education and Clinical Center.

Funding Source: This work was directly supported by National Institutes of Health - National Institute of Allergy and Infectious Diseases grant K08AI112506 (Dr. Logan) and grants R01AI072219, R01AI063517, and R01AI100560 (Dr. Bonomo). This work was also supported by the Department of Veterans Affairs Research and Development under award number I01BX001974, VISN 10 Geriatrics Research, Education and Clinical Center (Dr. Bonomo).

\section{References}

1. Logan LK, Braykov NP, Weinstein RA, and Laxminarayan R 2014 Extended-Spectrum BLactamase-Producing and Third-Generation Cephalosporin-Resistant Enterobacteriaceae in Children: Trends in the United States, 1999-2011. Journal of the Pediatric Infectious Diseases Society. 3:320-28. doi: 10.1093/jpids/piu010. [PubMed: 26625452]

2. Logan LK, Renschler JP, Gandra S, Weinstein RA, Laxminarayan R, Centers for Disease Control, and Prevention Epicenters Program. 2015 Carbapenem-Resistant Enterobacteriaceae in Children, United States, 1999-2012. Emerg. Infect. Dis. 21:2014-2021. doi: 10.3201/eid2111.150548 [doi]. [PubMed: 26486124]

3. Lukac PJ, Bonomo RA, and Logan LK 2015 Extended-spectrum $\beta$-lactamase-producing Enterobacteriaceae in children: old foe, emerging threat. Clinical Infectious Diseases. 60:13891397. [PubMed: 25595742]

4. Logan LK, and Weinstein RA 2017 The Epidemiology of Carbapenem-Resistant Enterobacteriaceae: The Impact and Evolution of a Global Menace. J. Infect. Dis. 215:S28-S36. [PubMed: 28375512]

5. Cantón R, and Coque TM 2006 The CTX-M $\beta$-lactamase pandemic. Curr. Opin. Microbiol. 9:466475. [PubMed: 16942899]

6. Robicsek A, Jacoby GA, and Hooper DC 2006 The worldwide emergence of plasmid-mediated quinolone resistance. The Lancet Infectious Diseases. 6:629-640. [PubMed: 17008172]

7. Medernach RL, and Logan LK 2018 The Growing Threat of Antibiotic Resistance in Children. Infect. Dis. Clin. North Am. 32:1-17. [PubMed: 29406971]

8. Clinical and Laboratory Standards Institute. 2010 Performance Standards for Antimicrobial Susceptibility Testing: Twentieth Informational Supplement (June 2010 Update). 12 29, 2011.

9. Centers for Disease Control and Prevention (CDC). CDC - 2012 CRE Toolkit - Guidance for Control of Carbapenem-resistant Enterobacteriaceae (CRE). 2015 Available at: http:// www.cdc.gov/hai/pdfs/cre/cre-guidance-508.pdf

10. Centers for Disease Control and Prevention. 2013 Modified Hodge Test for Carbapenemase Detection in Enterobacteriaceae. .

11. Bogaerts P, Hujer AM, Naas T, de Castro RR, Endimiani A, Nordmann P, Glupczynski Y, and Bonomo RA 2011 Multicenter evaluation of a new DNA microarray for rapid detection of clinically relevant bla genes from beta-lactam-resistant gram-negative bacteria. Antimicrob. Agents Chemother. 55:4457-4460. doi: 10.1128/AAC.00353-11 [doi]. [PubMed: 21746960]

12. Cunningham S, Johnston B, Vasoo S, Johnson J, and Patel R 2014 Abstr. Evaluation of CheckPoints Check-MDR CT103 PCR-Microarray Kit for Detection and Classification of ESBL, AmpC and Carbapenemase Genes 54th Interscience Conference on Antimicrobial Agents and Chemotherapy. Washington, D.C.

13. Logan LK, Hujer AM, Marshall SH, Domitrovic TN, Rudin SD, Zheng X, Qureshi NK, Hayden MK, Scaggs FA, Karadkhele A, and Bonomo RA 2016 Analysis of beta-Lactamase Resistance Determinants in Enterobacteriaceae from Chicago Children: a Multicenter Survey. Antimicrob. Agents Chemother. 60:3462-3469. doi: 10.1128/AAC.00098-16 [doi]. [PubMed: 27021322]

14. Hujer KM, Hujer AM, Endimiani A, Thomson JM, Adams MD, Goglin K, Rather PN, Pennella TT, Massire C, Eshoo MW, Sampath R, Blyn LB, Ecker DJ, and Bonomo RA 2009 Rapid 
determination of quinolone resistance in Acinetobacter spp. J. Clin. Microbiol. 47:1436-1442. [PubMed: 19297590]

15. Endimiani A, Hujer AM, Perez F, Bethel CR, Hujer KM, Kroeger J, Oethinger M, Paterson DL, Adams MD, Jacobs MR, Diekema DJ, Hall GS, Jenkins SG, Rice LB, Tenover FC, and Bonomo RA 2009 Characterization of blaKPC-containing Klebsiella pneumoniae isolates detected in different institutions in the Eastern USA. J. Antimicrob. Chemother. 63:427-437. [PubMed: 19155227]

16. Jacoby GA 2017 Plasmid-mediated quinolone resistance, p. 265-268. In Anonymous Antimicrobial Drug Resistance. Springer.

17. Bingen-Bidois M, Clermont O, Bonacorsi S, Terki M, Brahimi N, Loukil C, Barraud D, and Bingen E 2002 Phylogenetic analysis and prevalence of urosepsis strains of Escherichia coli bearing pathogenicity island-like domains. Infect. Immun. 70:3216-3226. [PubMed: 12011017]

18. Carattoli A, Bertini A, Villa L, Falbo V, Hopkins KL, and Threlfall EJ 2005 Identification of plasmids by PCR-based replicon typing. J. Microbiol. Methods. 63:219-228. doi: 10.1016/ j.mimet.2005.03.018. [PubMed: 15935499]

19. Centers for Disease Control and Prevention (CDC). 2017 The National Healthcare Safety Network (NHSN) Patient Safety Component Manual.

20. Canton R, Gonzalez-Alba JM, and Galan JC 2012 CTX-M Enzymes: Origin and Diffusion. Front. Microbiol. 3:110. doi: 10.3389/fmicb.2012.00110 [doi]. [PubMed: 22485109]

21. Zerr DM, Qin X, Oron AP, Adler AL, Wolter DJ, Berry JE, Hoffman L, and Weissman SJ 2014 Pediatric infection and intestinal carriage due to extended-spectrum-cephalosporin-resistant Enterobacteriaceae. Antimicrob. Agents Chemother. 58:3997-4004. doi: 10.1128/AAC.02558-14 [doi]. [PubMed: 24798269]

22. Lin MY, Lyles-Banks RD, Lolans K, Hines DW, Spear JB, Petrak R, Trick WE, Weinstein RA, Hayden MK, and Centers for Disease Control and Prevention Epicenters Program. 2013 The importance of long-term acute care hospitals in the regional epidemiology of Klebsiella pneumoniae carbapenemase-producing Enterobacteriaceae. Clinical Infectious Diseases. 57:12461252. [PubMed: 23946222]

23. Strysko JP, Mony V, Cleveland J, Siddiqui H, Homel P, and Gagliardo C 2016 International travel is a risk factor for extended-spectrum $\beta$-lactamase-producing Enterobacteriaceae acquisition in children: A case-case-control study in an urban US hospital. Travel Medicine and Infectious Disease. 14:568-571. [PubMed: 27890813]

24. Islam S, Selvarangan R, Kanwar N, McHenry R, Chappell JD, Halasa N, Wikswo ME, Payne DC, Azimi PH, McDonald LC, and Gomez-Duarte OG 2017615 Intestinal Carriage of ThirdGeneration Cephalosporin-Resistant and Extended-Spectrum beta-Lactamase-Producing Enterobacteriaceae in Healthy US Children. Journal of the Pediatric Infectious Diseases Society. 7:234-40.

25. Laupland KB, Church DL, Vidakovich J, Mucenski M, and Pitout JD 2008 Community-onset extended-spectrum $\beta$-lactamase (ESBL) producing Escherichia coli: importance of international travel. J. Infect. 57:441-448. [PubMed: 18990451]

26. Logan LK, Meltzer LA, McAuley JB, Hayden MK, Beck T, Braykov NP, Laxminarayan R, and Weinstein RA 2014 Extended-Spectrum $\beta$-Lactamase-Producing Enterobacteriaceae Infections in Children: A Two-Center Case-Case-Control Study of Risk Factors and Outcomes in Chicago, Illinois. Journal of the Pediatric Infectious Diseases Society. 3:312-319. [PubMed: 26625451]

27. Silbergeld EK, Graham J, and Price LB 2008 Industrial food animal production, antimicrobial resistance, and human health. Annu. Rev. Public Health. 29:151-169. [PubMed: 18348709]

28. Riley LW, Raphael E, and Faerstein E 2013 Obesity in the United States - dysbiosis from exposure to low-dose antibiotics? Front. Public. Health. 1:69. doi: 10.3389/fpubh.2013.00069 [doi]. [PubMed: 24392444]

29. Steinberger J, Daniels SR, American Heart Association Atherosclerosis, Hypertension, and Obesity in the Young Committee (Council on Cardiovascular Disease in the Young), and American Heart Association Diabetes Committee (Council on Nutrition, Physical Activity, and Metabolism). 2003 Obesity, insulin resistance, diabetes, and cardiovascular risk in children: an American Heart Association scientific statement from the Atherosclerosis, Hypertension, and Obesity in the Young Committee (Council on Cardiovascular Disease in the Young) and the Diabetes Committee 
(Council on Nutrition, Physical Activity, and Metabolism). Circulation. 107:1448-1453. [PubMed: 12642369]

30. Hansen LH, Sørensen SJ, Jørgensen HS, and Jensen LB 2005 The prevalence of the OqxAB multidrug efflux pump amongst olaquindox-resistant Escherichia coli in pigs. Microbial Drug Resistance. 11:378-382. [PubMed: 16359198]

31. Goyal MK, Johnson TJ, Chamberlain JM, Casper TC, Simmons T, Alessandrini EA, Bajaj L, Grundmeier RW, Gerber JS, Lorch SA, Alpern ER, and Pediatric Care Applied Research Network (PECARN). 2017 Racial and Ethnic Differences in Antibiotic Use for Viral Illness in Emergency Departments. Pediatrics. doi: e20170203 [pii].

32. Popovich KJ, Weinstein RA, Aroutcheva A, Rice T, and Hota B 2010 Community-associated methicillin-resistant Staphylococcus aureus and HIV: intersecting epidemics. Clinical Infectious Diseases. 50:979-987. [PubMed: 20192731] 
TABLE 1

CHARACTERISTICS OF FQR AND PMFQR ENTEROBACTERIACEAE

\begin{tabular}{|c|c|c|}
\hline Variable $^{a}$ & FQR Ent & PMFQR Ent \\
\hline Patient & $\mathrm{n}=82$ & $\mathrm{n}=53$ \\
\hline \multicolumn{3}{|l|}{ Organism $b$} \\
\hline E. coli & $65(79.3)$ & $40(75.5)$ \\
\hline Klebsiella sp. & $7(8.5)$ & $7(13.2)$ \\
\hline Proteus sp. & $7(8.5)$ & $4(7.5)$ \\
\hline Enterobacter $s p$ & $3(3.7)$ & $2(3.8)$ \\
\hline Other & $0(0)$ & $0(0)$ \\
\hline \multicolumn{3}{|l|}{ Source } \\
\hline Urine & $58(70.7)$ & $37(69.8)$ \\
\hline Respiratory & $12(14.6)$ & $7(13.2)$ \\
\hline Abscess/Wound & $4(4.9)$ & $3(5.7)$ \\
\hline Blood & $3(3.7)$ & $3(5.7)$ \\
\hline Peritoneal/Abdomen & $2(2.4)$ & $1(1.9)$ \\
\hline Central Nervous System & $1(1.2)$ & $1(1.9)$ \\
\hline Other & $2(2.4)$ & $1(1.9)$ \\
\hline \multicolumn{3}{|l|}{ Co-Antibiotic Resistance } \\
\hline Trimethoprim/Sulfamethoxazole & $59(72.0)$ & $38(71.7)$ \\
\hline Gentamicin & $44(53.7)$ & $35(66.0)$ \\
\hline Amikacin & $3(3.7)$ & $3(5.7)$ \\
\hline Carbapenem & $1(1.2)$ & $1(1.9)$ \\
\hline \multicolumn{3}{|l|}{ Blagene association ${ }^{c}$} \\
\hline CTX-M-1 $1_{\text {group }}$ & $51(62.2)$ & $42(79.2)$ \\
\hline CTX-M- $9_{\text {group }}$ & $13(15.9)$ & $3(5.7)$ \\
\hline $\mathrm{SHV}_{\mathrm{ESBL}}$ & $9(11.0)$ & $6(11.3)$ \\
\hline $\mathrm{VEB}_{\mathrm{ESBL}}$ & $1(1.2)$ & $1(1.9)$ \\
\hline $\mathrm{CMY}_{\mathrm{AmpC}}$ & $2(2.4)$ & $1(1.9)$ \\
\hline $\mathrm{ACT} / \mathrm{MIR}_{\mathrm{AmpC}}$ & $4(4.9)$ & $1(1.9)$ \\
\hline $\mathrm{KPC}_{\mathrm{CRE}}$ & $1(1.2)$ & $1(1.9)$ \\
\hline Mutation in $\mathrm{QRDR}^{d}$ & $71(89.9)$ & $43(87.8)$ \\
\hline Phylogenetic group of E. coli & $\mathrm{n}=65$ & $\mathrm{n}=40$ \\
\hline B2 & $49(75.4)$ & $31(77.5)$ \\
\hline $\mathrm{D}$ & $11(16.9)$ & $6(15.0)$ \\
\hline A & $4(6.1)$ & $3(7.5)$ \\
\hline B1 & $1(1.5)$ & $0(0)$ \\
\hline
\end{tabular}

Pediatr Infect Dis J. Author manuscript; available in PMC 2020 June 01. 
${ }^{a}$ Values represent n (\%). Abbreviations: Ent, Enterobacteriaceae, FQR, Flouroquinolone resistant; PMFQR, Plasmid-mediated Fluoroquinolone resistant

$b_{\text {One isolate studied per patient }}$

Isolates may harbor one or more bla gene.

$d_{3}$ of the 82 isolates did not yield a result, calculation based on 79 isolates. 
TABLE 2.

Multivariable Analysis of Factors Associated With PMFQR Enterobacteriaceae Infections in Children

\begin{tabular}{l|c|c|c|}
\hline Associated Factor with PMFQR infection $^{a}$ & OR & 95\% CI & p value \\
\hline Outpatient Clinic Location at time of infection diagnosis & 33.9 & $7.08,162.8$ & $<0.001$ \\
\hline Race not white, black or Hispanic & 6.5 & $1.70,24.3$ & 0.006 \\
\hline Home Residence in Southwest Region (SW Chicago and SW Suburbs) & 5.6 & $1.6,19.2$ & 0.006 \\
\hline Home Residence in Downtown Region (Near North Side, Loop, Northside) & 0.03 & $0.002,0.33$ & 0.005 \\
\hline \\
$\begin{array}{l}\text { Abbreviations, SW, Southwest; Loop, Chicago Loop. } \\
b_{\text {Reference Region, West Region. }}\end{array}$
\end{tabular}

